\title{
COMUNICAÇÃO BREVE Relato de caso de síndrome de Hikikomori em Portugal: uma síndrome primária ou expressão de outra perturbação mental?
}

\author{
Case report of Hikikomori syndrome in Portugal: a primary \\ syndrome or the expression of another mental disorder? \\ Ana Margarida Mota' \\ https://orcid.org/0000-0002-3351-6295 \\ Mariana Lázaro? \\ https://orcid.org/0000-0001-6742-3882 \\ Elsa Gaspar' \\ https://orcid.org/0000-0001-5301-1764 \\ David Rosa' \\ https://orcid.org/0000-0002-5767-5602 \\ Ana Cristina Farias ${ }^{1}$ \\ https://orcid.org/0000-0002-0942-1237
}

\section{Palavras-chave}

Hikikomori, isolamento

social, hikikomori

secundário.

\section{Keywords}

Hikikomori, social

withdrawal, secondary

hikikomori.

\section{RESUMO}

O fenómeno hikikomori, forma de isolamento social grave e prolongado, constitui um desafio diagnóstico. A nível conceptual, alguns autores diferenciam o hikikomori em primário e secundário. Este último conceito é utilizado quando está presente comorbilidade que, pelo menos parcialmente, explique a síndrome, enquanto na ausência de diagnóstico psiquiátrico denomina- se "hikikomori primário". Os autores apresentam um caso clínico português com as mesmas características sociodemográficas e clínicas descritas no Japão. Esse caso ilustra que o hikikomori pode potencialmente ser explicado por uma perturbação psiquiátrica subjacente, nem sempre clara à apresentação, e a importância da avaliação cuidadosa e continuada no tempo, por forma a conseguir o esclarecimento diagnóstico e intervenção terapêutica adequada.

\begin{abstract}
Diagnosing hikikomori, a form of severe and prolonged social withdrawal, is challenging. Conceptually speaking, some authors differentiate "primary" and "secondary" hikikomori. The latter term has been used when there is co-morbidity that at least partially explains the syndrome, whereas in the absence of a psychiatric diagnosis it is called "primary hikikomori". The authors present a Portuguese case report with the same sociodemographic and clinical characteristics described in Japan. This case illustrates that hikikomori can potentially be explained by an underlying psychiatric disorder that is not always clear at the presentation and the importance of careful and continuous assessment, in order to achieve diagnostic clarification and adequate therapeutic intervention.
\end{abstract}

1 Centro Hospitalar Psiquiátrico de Lisboa, Portugal.

Recebido em

9/Jul/2019

Aprovado em

13/Ago/2019

DOI: 10.1590/0047-2085000000243

Endereço para correspondência: Ana Margarida Mota

Avenida do Brasil 53

1749-002 - Lisboa, Portugal

E-mail:mota_margarida@hotmail.com 


\section{INTRODUÇÃO}

Nos últimos anos, o isolamento social grave tem-se tornado um importante problema clínico, social e de saúde pública!. Hikikomori (do japonês "afastamento"2 ou "pessoa isolada"3) é um fenómeno em que indivíduos se isolam nas suas casas, evitando várias situações sociais (como ir à escola e ao trabalho, interagir socialmente fora de casa etc.), durante pelo menos seis meses ${ }^{4,5}$. É mais comum no sexo masculino e tipicamente se manifesta na adolescência ou início da idade adulta $^{6}$. A sua natureza é multifactorial e não se encontra totalmente esclarecida, mas frequentemente se associa a factores como: temperamento tímido, experiências traumáticas na infância (principalmente bullying), dificuldades académicas e/ou laborais e dinâmicas familiares disfuncionais 2,6-8.

A síndrome de hikikomori foi inicialmente descrita no Japão por Tomita Fujiya, na década de 1980, e considerada um problema ligado à cultura japonesa (japanese culture-bound syndrome $)^{2,9}$. No entanto, nos últimos anos, tornou-se uma preocupação internacional pelo número crescente de casos relatados fora do Japão, sendo conhecidos casos na Europa, América e noutros países asiáticos ${ }^{1-3,6,8,10-14}$

O diagnóstico dessa condição é desafiante dada a ausência de critérios de diagnóstico consensuais e a elevada prevalência de perturbação psiquiátrica comórbida (como perturbações depressivas, de ansiedade, da personalidade, do espectro do autismo, psicóticas e de adição à internet/ videojogos) $)^{2,8,15}$.

Vários autores defendem a diferenciação em hikikomori primário e secundário. No hikikomori primário considera-se que $\mathrm{o}$ isolamento social resulta da interacção entre os indivíduos, família e sociedade, e não é um sintoma de doença psiquiátrica 16,17. Por sua vez, o conceito "hikikomori secundário" deve ser utilizado se estiver presente perturbação mental que, pelo menos parcialmente, explique a síndrome ${ }^{28,15}$.

A somar às dificuldades diagnósticas, a adolescência e o início da vida adulta representam um período de transição e de início de várias perturbações psiquiátricas, em que tipicamente a sintomatologia inicial é insidiosa e inespecífica, tal como o isolamento social'5.

\section{CASO CLÍNICO}

O caso clínico apresentado refere-se a um jovem de 18 anos, do sexo masculino, solteiro, que reside com os pais e o irmão gémeo em zona suburbana e que, durante cerca de um ano e meio, se isolou voluntariamente em casa após ter reprovado no $10^{\circ}$ ano, deixando a turma do irmão gémeo.

Durante o período que permaneceu isolado em casa, maioritariamente no quarto, saiu apenas uma vez para visitar a avó que estava hospitalizada. Apresentava inversão do ciclo de sono- vigília, ocupando as noites a ver televisão ou a utilizar o computador (a jogar e a navegar na internet), dormindo durante o dia. Não demonstrava qualquer interesse em retomar a escola ou actividades sociais, nem manifestava interesses particulares ou preferenciais por qualquer actividade.

Apresentava história de ter sido vítima de bullying na adolescência e temperamento tímido. Não tinha outros antecedentes relevantes, nomeadamente psiquiátricos, médico-cirúrgicos, ou história de uso de tabaco, álcool ou de substâncias ilícitas. Sem história de perturbação psiquiátrica na família, e o irmão gémeo era saudável e iria concluir o ensino secundário nesse ano. Do ambiente familiar, destacamos o facto de o pai estar ausente durante períodos prolongados por motivos laborais e de a mãe revelar dificuldade em estabelecer autoridade e impôr limites. Apresentavam condição socioeconómica confortável.

A mãe procurou ajuda médica para o filho, dado estar preocupada por ele não sair de casa e ter aumentado muito de peso (cerca de $25 \mathrm{~kg}$ ). Por recusa do jovem em recorrer aos serviços de saúde, foi realizada visita domiciliária pela equipa de saúde mental local. Nessa visita, apresentava-se tenso, hostil, não estabelecia contacto visual e não colaborava na entrevista, impossibilitando a exclusão de perturbação mental grave. Por esse motivo, foi requerida uma avaliação clínico-psiquiátrica ao abrigo da lei de saúde mental portuguesa, da qual resultou internamento.

No internamento em serviço de agudos, o doente apresentava-se pouco colaborante, apurando-se apenas quadro clínico caracterizado por anedonia, abulia, adinamia, isolamento social e negligência do autocuidado. Inicialmente, no exame do estado mental, destacavam-se hipomímia, ligeira lentificação psicomotora e contacto distante; discurso provocado, de conteúdo pobre e aprosódico; humor eutímico com diminuição da reactividade emocional; pensamento sem alterações formais, não sendo possível avaliar adequadamente o seu conteúdo pela pouca colaboração; não se apuravam alterações da percepção; não apresentava insight para as alterações psicopatológicas e necessidade de tratamento. Na enfermaria, interagia pouco com os doentes e profissionais de saúde, mantendo postura reservada.

A equipa médica que o acompanhou no internamento considerou, como hipótese diagnóstica mais provável, fase prodrómica de perturbação psicótica, tendo como principais manifestações isolamento social grave e ruptura do funcionamento prévio. Foi medicado com palideridona (titulada até $12 \mathrm{mg} / \mathrm{dia}$ ), tendo tido alta após 31 dias. À data de alta, apresentava-se mais interativo e colaborante, aceitando a transição para internamento em hospital de dia.

Por sua vez, no hospital de dia, considerou-se que o quadro clínico seria mais bem explicado por um quadro depressivo, decidindo-se pela redução, e posterior suspensão, da paliperidona e introdução de terapêutica antidepressiva com bupropiona. O doente apresentou melhoria significativa, tornando-se progressivamente mais colaborante, o que 
permitiu esclarecer que o diagnóstico que esteve na origem do isolamento social e do absentismo e evitamento escolares foi antes o de uma perturbação de ansiedade social e que a sintomatologia depressiva se manifestou posteriormente. Foi alterado o antidepressivo para sertralina, com titulação progressiva até 200 mg/dia, tendo-se alcançado remissão total dos sintomas ao fim de dois meses e meio. Simultaneamente, teve acompanhamento psicoterapêutico cognitivo-comportamental individual e de grupo, além de ter sido realizada intervenção psicoeducativa familiar. Teve alta ao fim de 99 dias, com o projecto pós-alta de retomar os estudos por meio do ensino recorrente (alternativa para adultos concluírem o ensino secundário). Iniciou a prática de desporto (jogging e futebol com um grupo de amigos) e teve acompanhamento em consulta de nutrição, conseguindo perda de peso significativa. Compareceu regularmente em consultas de psiquiatria de ambulatório, tendo sido mantida a terapêutica com sertralina.

Após um ano de seguimento o doente mantém melhoria clínica, sendo referida apenas ansiedade em situações de exposição social, especialmente em contexto escolar (por exemplo, apresentação oral de trabalhos). No entanto, essas queixas não têm impacto significativo, estando bem integrado social e academicamente.

\section{DISCUSSÃO}

Os contextos cultural, social e histórico do doente influenciam a forma de apresentação da perturbação psiquiátrica. Foi sugerido que o contexto socioeconómico e cultural do Japão possa contribuir para o aparecimento do fenómeno hiki$k_{0}$ ori ${ }^{18}$. No entanto, os casos relatados em diferentes partes do mundo 1-3,6,8,10-14, bem como o caso português que descrevemos, apresentam características clínicas e sociodemográficas semelhantes às descritas no Japão, reforçando que essa síndrome não está unicamente ligada à cultura japonesa.

Nos últimos anos tem sido discutido se o hikikomori é uma nova perturbação psiquiátrica ou a manifestação de uma perturbação mental preexistente. Estudos prévios sugerem que o hikikomori tem elevada comorbilidade com outras perturbações psiquiátricas ( $33 \%$ a $54,5 \%)^{7,16}$ e dados mais recentes indiciam que o diagnóstico comórbido pode ser ainda mais frequente do que foi inicialmente considerado ${ }^{8,19}$.

O diagnóstico diferencial entre o fenómeno hikikomorie a fase inicial de outras perturbações mentais é particularmente difícil, podendo o isolamento social grave ser a manifestação clínica inicialmente observada em múltiplos quadros psiquiátricos. Esse fenômeno tem sido particularmente associado à adição à internet/videojogos, que é um diagnóstico psiquiátrico que surgiu recentemente, ainda alvo de grande debate, e cujos critérios de diagnóstico não estão bem estabelecidos ${ }^{2,15}$. O diagnóstico diferencial entre essas duas condições é complexo, tendo em conta que compartilham a falta de interesse pela escola e trabalho, e as dificuldades nos relacionamentos interpessoais. No caso apresentado, o uso de internet e jogos on-line parece ter surgido secundariamente ao isolamento social, não representava uma preocupação central na vida do doente e não se apuraram sintomas de abstinência. Apesar disso, consideramos que este é um diagnóstico diferencial a considerar.

O hikikomori primário pode ocorrer, mas, tal como alguns autores têm defendido, será menos frequente. Ainda que um estudo $^{16}$ descreva que numa população do Japão cerca de metade dos casos de hikikomori não apresentavam perturbação psiquiátrica, defendemos que os quadros de hikikomori podem potencialmente ser explicados por uma perturbação psiquiátrica subjacente, que nem sempre é clara à apresentação, tal como ilustrado no caso clínico que apresentámos.

Com este caso pretendemos ainda destacar a importância de uma avaliação cuidadosa e continuada no tempo para esclarecer o diagnóstico e dirigir a intervenção terapêutica. Consideramos que um diagnóstico prematuro de Hikikomori primário pode, assim, comprometer a intervenção, prejudicando o prognóstico do doente.

A relutância dos doentes em procurar cuidados de saúde representa outro factor condicionante do prognóstico, uma vez que esses doentes frequentemente não consideram o isolamento social como problemático².

Provavelmente a prevalência de hikikomori tem sido subestimada ${ }^{3}$, sendo o primeiro obstáculo identificar esses indivíduos e encaminhá-los para o tratamento². Assim, a visita domiciliária pode ser a única forma de identificar e tratar esses doentes em que o isolamento social se apresenta como o sintoma principal e dominante do quadro clínico ${ }^{23,8}$.

\section{CONCLUSÃO}

O hikikomori não deve ser considerado um fenómeno cultural japonês, dado o crescente número de casos relatados internacionalmente.

Provavelmente a prevalência de hikikomori tem sido subestimada, por falta de conhecimento dos profissionais de saúde acerca desse tema e pela dificuldade em chegar a esses doentes.

No futuro, é importante encontrar critérios consensuais para o diagnóstico de hikikomori, bem como investigar a associação entre essa e outras perturbações mentais, por forma a desenvolver estratégias de prevenção e tratamento eficazes.

\section{CONTRIBUIÇÕES INDIVIDUAIS}

Ana Margarida Mota e Mariana Lázaro - Observaram o doente, elaboraram a história clínica e fizeram a revisão da literatura, contribuindo de igual forma para o artigo. 
Elsa Gaspar - Observou o doente e contribuiu para a concepção e revisão do artigo.

David Rosa - Realizou a formatação e a revisão do artigo.

Ana Cristina Farias - Realizou a supervisão e a revisão do artigo.

\section{CONFLITO DE INTERESSES}

Nada a declarar.

\section{REFERÊNCIAS}

1. Kato T, Kanba S, Teo A. Hikikomori: experience in Japan and international relevance. World Psychiatry. 2018;17(1);105-6.

2. Domingues-Castro M, Torres A. Hikikomori: revisão sobre um grave fenômeno de isolamento social. J Bras Psiquiatr. 2018;68(4):264-72.

3. Lee $Y$, Lee J, Choi T, Choi J. Home visitation program for detecting, evaluating and treating socially withdrawn youth in Korea. Psychiatry Clin Neurosci. 2013;67(4):193-202.

4. Saito K. Hikikomori no hyouka-shien Ni kansaru gaido-rain [guideline of hikikomori for their evaluations and supports]. Tokyo: Ministry of Health, Labour and Welfare; 2010.

5. Tateno M, Park T, Kato T, Umene-Nakano W. Hikikomori as a possible clinical term in Psychiatry: a questionnaire survey. BMC Psychiatry. 2012;12:169.

6. Teo A, Chen J, Kubo H, Katsuki R, Sato-Kasai M, Shimokawa N, et al. Development and validation of the 25-item Hikikomori Questionnaire (HQ-25). Psychiatry Clin Neurosci. 2018;72(10):780-8.
7. Teo A. A new form of social withdrawal in Japan: a review of hikikomori. Int J Soc Psychiatry. 2010;56(2):178-85

8. Malagón-Amor Á, Córcoles-Martínez D, Martín-López L, Pérez-Solà V. Hikikomori in Spain: A descriptive study. Int J Soc Psychiatry. 2015;61(5):475-83.

9. Nonaka S, Shimada H, Sakai M. Characteristics of family interaction of individuals with Hikikomori (prolonged social withdrawal) from the viewpoint of behavior theory. Japanese Psychological Research. Jap Psychol Assoc. 2018.

10. Chauliac N, Couillet A, Faivre S, Brochard N, Terra JL. Characteristics of socially withdrawn youth in France: a retrospective study. Int J Soc Psychiatry. 2017.

11. Gondim F, Aragão A, Holanda Filha J, Messias E. Hikikomori in Brazil: 29 years of voluntary social withdrawal. Asian J Psychiatry. 2017;30:163-4.

12. García-Campayo J, Alda M, Sobradiel N, Abós B. Un caso de hikikomori en España. Med Clin (Barc). 2007;129(8):318-9.

13. Ovejero S, Caro-Cañizares I, León-Martinez V, Baca-Garcia E. Prolonged social withdrawal disorder: a hikikomori case in Spain. Int J Soc Psychiatry. 2014;60(6):562-5.

14. Wu A, Ooi J, Wong P, Catmur C, Lau J. Evidence of pathological social withdrawal in nonAsian countries: a global health problem? Lancet Psychiatry. 2019;6:195-6.

15. Stip E, Thibault A, Beauchamp-Chatel A, Kisely S. Internet addiction, hikikomori syndrome, and the prodromal phase of psychosis. Front Psychiatry. 2016;7:6.

16. Koyama A, Miyake Y, Kawakami N, Tsuchiya M, Tachimori H, Takeshima T. Lifetime prevalence, psychiatric comorbidity and demographic correlates of 'hikikomori' in a community population in Japan. Psychiatry Res. 2010;176(1):69-74.

17. Teo A. Social isolation associated with depression: A case report of hikikomori. Int J Soc Psychiatry. 2013;59(4):339-41.

18. Kato T, Tateno M, Shinfuku N, Fujisawa D, Teo A, Startorius N, et al. Does the 'hikikomori' syndrome of social withdrawl exist outside Japan?: A preliminary international investigation. Soc Psychiatry Epidemiol. 2012;47(7):1061-75.

19. Kondo N, Sakai M, Kuroda Y, Kiyota Y, Kitabata Y, Kurosawa M. General condition of hikikomori (prolonged social withdrawal) in Japan: Psychiatric diagnosis and outcome in mental health welfare centres. Int J Soc Psychiatry. 2013;59(1):79-86. 